\title{
31. Using IF .. ELSE (2)
}

Groups of statements (including further IF..ELSE statements) may be carried out following IF or ELSE commands:

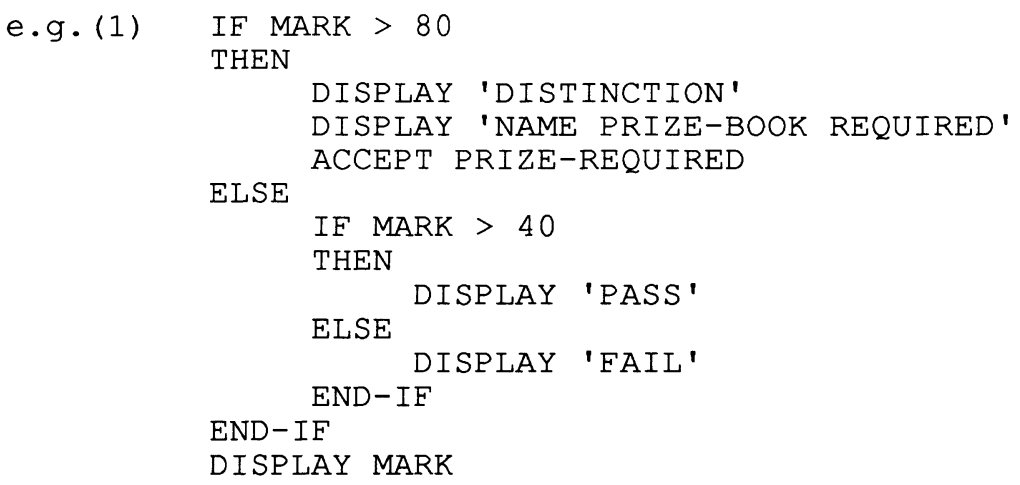

For any mark over 80 the computer will display 'Distinction' and ask what prize the student would like; marks lower than this are divided into passes and fails. The second END-IF indicates that the IF .. ELSE sentence has finished - so the statement DISPLAY-MARK applies to all students and every mark is printed.

NOTE THAT THE INDENTATION HAS NO EFFECT - (It just makes the program easier to understand). THE EFFECT OF THE SENTENCE IS GOVERNED BY THE RULE - each END-IF is paired with the most recent IF that has not already been terminated.

e.g. (2)

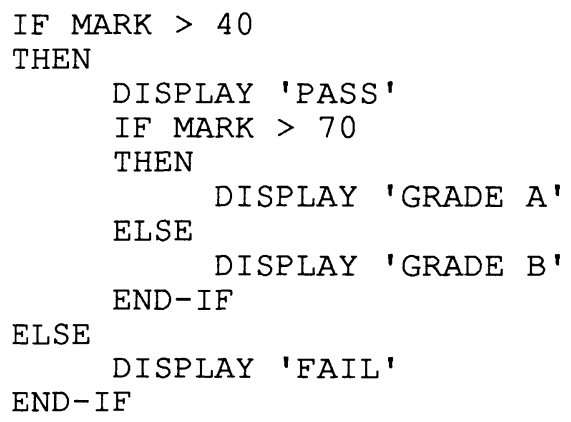

\section{Exercises}

1. In Example 2 above, what would be displayed on the screen for each of
the following marks ?
a) 40 b) 41
c) 70
d) 75
e) 10 f) 60

2. Write a COBOL sentence which will carry out the instructions in the following decision table:

\begin{tabular}{llllll}
\hline CONDITIONS: & & & & \\
English Mark >=50 & Y & Y & N & N \\
French Mark $>=50$ & Y & N & Y & N \\
\hline ACTIONS: & & & & \\
Display 'credit' & $\mathrm{X}$ & & & \\
Display 'pass' & & $\mathrm{X}$ & $\mathrm{X}$ & \\
Display 'fail'' & & & & $\mathrm{X}$ \\
\hline
\end{tabular}

